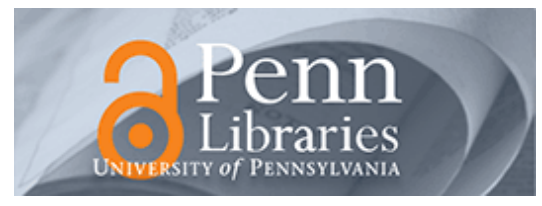

University of Pennsylvania

ScholarlyCommons

7-21-2009

\title{
Quasi-Two-Dimensional Diffusion of Single Ellipsoids: Aspect Ratio and Confinement Effects
}

\author{
Yilong Han \\ Hong Kong University of Science and Technology; University of Pennsylvania \\ Ahmed Alsayed \\ University of Pennsylvania \\ Maurizio Nobili \\ University Montpellier II \\ Arjun G. Yodh \\ University of Pennsylvania, yodh@phsyics.upenn.edu
}

Follow this and additional works at: https://repository.upenn.edu/physics_papers

Part of the Physics Commons

\section{Recommended Citation}

Han, Y., Alsayed, A., Nobili, M., \& Yodh, A. G. (2009). Quasi-Two-Dimensional Diffusion of Single Ellipsoids: Aspect Ratio and Confinement Effects. Retrieved from https://repository.upenn.edu/physics_papers/62

\footnotetext{
Suggested Citation:

Han, Y., A. Alsayed, M. Nobili, and A.G. Yodh. (2009). "Quasi-two-dimensional diffusion of single ellipsoids: Aspect ratio and confinement effects." Physical Review E. 80, 011403.

(c) 2009 The American Physical Review

http://dx.doi.org/10.1103/PhysRevE.80.011403.

This paper is posted at ScholarlyCommons. https://repository.upenn.edu/physics_papers/62

For more information, please contact repository@pobox.upenn.edu.
} 


\title{
Quasi-Two-Dimensional Diffusion of Single Ellipsoids: Aspect Ratio and Confinement Effects
}

\author{
Abstract \\ We report on video-microscopy measurements of the translational and rotational Brownian motions of \\ isolated ellipsoidal particles in quasi-two-dimensional sample cells of increasing thickness. The long-time \\ diffusion coefficients were measured along the long $\left(D_{a}\right)$ and short $\left(D_{b}\right)$ ellipsoid axes, respectively, and \\ the ratio, $D_{a} / D_{b}$, was determined as a function of wall confinement and particle aspect ratio. In three \\ dimensions (3D), this ratio $\left(D_{a} / D_{b}\right)$ cannot be larger than 2 , but in quasi-two dimensions, wall \\ confinement was found to substantially alter diffusion anisotropy and substantially slow particle diffusion \\ along the short axis compared to 3D. \\ Disciplines \\ Physical Sciences and Mathematics | Physics

\section{Comments} \\ Suggested Citation: \\ Han, Y., A. Alsayed, M. Nobili, and A.G. Yodh. (2009). "Quasi-two-dimensional diffusion of single ellipsoids: \\ Aspect ratio and confinement effects." Physical Review E. 80, 011403. \\ (C) 2009 The American Physical Review \\ http://dx.doi.org/10.1103/PhysRevE.80.011403.
}




\title{
Quasi-two-dimensional diffusion of single ellipsoids: Aspect ratio and confinement effects
}

\author{
Yilong Han, ${ }^{1,2}$ Ahmed Alsayed, ${ }^{2,3}$ Maurizio Nobili, ${ }^{4}$ and Arjun G. Yodh ${ }^{2}$ \\ ${ }^{1}$ Hong Kong University of Science and Technology, Clear Water Bay, Hong Kong SAR, China \\ ${ }^{2}$ Department of Physics and Astronomy, University of Pennsylvania, 209 South 33rd Street, Philadelphia, Pennsylvania 19104, USA \\ ${ }^{3}$ Complex Fluid Lab, CNRS/Rhodia Joint Lab FRE3084, 350 George Patterson Drive, Bristol, Pennsylvania 19007, USA \\ ${ }^{4}$ Laboratoire des Colloides, Verres et Nanomateriaux, CNRS, University Montpellier II, Place E. Bataillon, 34090 Montpellier, France
}

(Received 24 February 2009; revised manuscript received 7 May 2009; published 21 July 2009)

\begin{abstract}
We report on video-microscopy measurements of the translational and rotational Brownian motions of isolated ellipsoidal particles in quasi-two-dimensional sample cells of increasing thickness. The long-time diffusion coefficients were measured along the long $\left(D_{a}\right)$ and short $\left(D_{b}\right)$ ellipsoid axes, respectively, and the ratio, $D_{a} / D_{b}$, was determined as a function of wall confinement and particle aspect ratio. In three dimensions (3D), this ratio $\left(D_{a} / D_{b}\right)$ cannot be larger than 2 , but in quasi-two dimensions, wall confinement was found to substantially alter diffusion anisotropy and substantially slow particle diffusion along the short axis compared to $3 \mathrm{D}$.
\end{abstract}

DOI: $10.1103 /$ PhysRevE.80.011403

PACS number(s): 82.70.Dd, 67.25.bf, 47.15.G-, 05.40.Jc

\section{INTRODUCTION}

In many biological and industrial processes, diffusing particles are often nonspherical and often move in confined geometries. Examples of such particles include proteins diffusing in membranes [1] and fine grains migrating through the pores of micro- and nanoporous media. To date, few quantitative measurements of anisotropic particle diffusion in confined geometries have been reported. However, new particle fabrication schemes and imaging technologies with novel image analysis tools now make direct measurement of the diffusion of anisotropic particles readily possible. In this contribution, we investigate anisotropic diffusion and hydrodynamic confinement effects associated with the motions of isolated ellipsoidal particles between two parallel plates. The paper expands upon related results presented cursorily in the supplementary online materials of a short recent report [2]. In particular, the problem is given historical and theoretical context, the experimental methods for measurement of particle confinement and tracking errors are described at length, the systematic effects of counter-ion screening and vertical displacements are assessed, and the anisotropy results are discussed at length and in the context of a simple qualitative picture.

The Brownian diffusion coefficient $D$ of an isolated spherical particle is well understood. It is inversely proportional to the drag (or friction) coefficient $\gamma$ via the Einstein relation

$$
D=k_{B} T / \gamma,
$$

where $k_{B}$ is the Boltzmann constant and $T$ is the temperature. For a prolate spheroid with long axis of length $2 a$ and two short axes of length $2 b$, translational diffusion is anisotropic and is described by diffusion coefficients $D_{a}=k_{B} T / \gamma_{a}$ along the long axis and $D_{b}=k_{B} T / \gamma_{b}$ along the short axes. The rotational diffusion coefficient of the prolate spheroid about its short axes is $D_{\theta}=k_{B} T / \gamma_{\theta}$. Generally, the drag coefficients $\gamma_{a}$, $\gamma_{b}$, and $\gamma_{\theta}$ depend on the shape and size of the ellipsoid. Brownian motion of anisotropic particles was first seriously considered by F. Perrin $[3,4]$, who computed these drag co- efficients analytically for a spheroid diffusing in three dimensions (3D). Interestingly, the ratio $D_{a} / D_{b}$ varies from 1 to 2 in $3 \mathrm{D}$, as the spheroid aspect ratio, $\phi=a / b$, varies from 1 to infinity.

The problem of diffusion in confined geometries, such as quasi-two-dimensional (2D) media, is different from the 3D case as a result of a complex interplay between hydrodynamic drag, the boundaries of the medium, and the particle geometry. Surfaces near a moving particle modify fluid flow fields, often increasing particle hydrodynamic drag. A full theoretical formulation of wall hydrodynamic effects has been developed for one sphere (or ellipsoid) coupled to one wall [5]. However, for more complicated situations such as a sphere or an ellipsoid confined by two parallel walls, the only available analytical solutions are for weak confinement in a few special symmetric configurations [5]. Recent numerical calculations [6], on the other hand, have been developed to derive the hydrodynamic drag of a single sphere and a linear chain of spheres confined more strongly in quasi-2D.

On the experimental side, the hydrodynamic drag of single spheres in weak confinement has been measured $[7,8]$ and video microscopy has been applied recently to measure anisotropic particle diffusion, including ellipsoids in quasi-2D [2] and 3D [9], colloidal clusters near one wall [10], and carbon nanotubes in weak confinement [11]. In the present contribution, we report measurements of hydrodynamic drag on ellipsoids in quasi-2D, confined between two parallel walls. We explore the strong confinement regime where drag coefficients are not readily available from theory and simulation, and we report on a light interference method to accurately measure the confinement. We find that the diffusion anisotropy is made stronger and the diffusion along ellipsoid short axes is dramatically slowed by wall confinement. Note that the present work examines how spatial confinement affects long-time scale traditional diffusion coefficients; this aim contrasts with the investigation of short-time scale time-dependent translation-rotation coupling and nonGaussian Brownian dynamics of a single ellipsoid, which was the focus of Ref. [2]. 


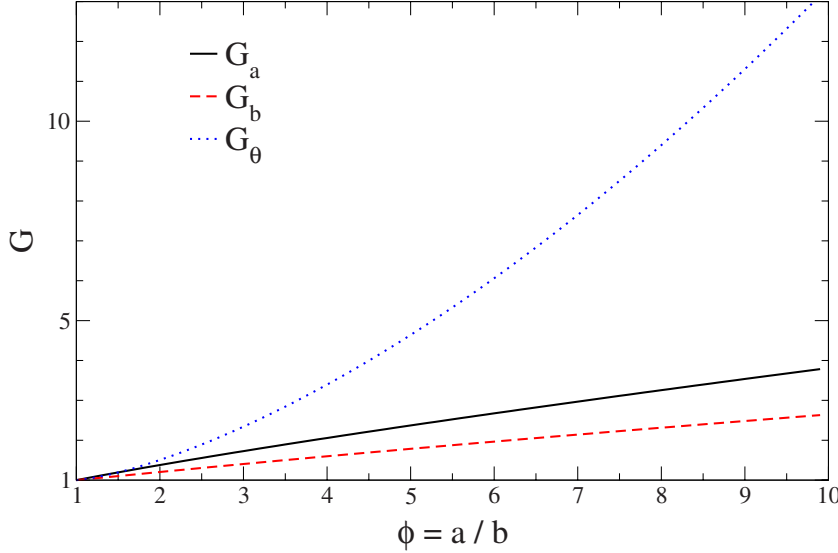

FIG. 1. (Color online) The geometric factors $G$ in Eq. (3) as a function of aspect ratio $\phi$.

\section{THEORY BACKGROUND}

When a spheroid with semiaxes $(a, b, b)$ moves along one of its principle axes with velocity $v$, through an unbounded quiescent fluid with viscosity $\eta$ at low Reynolds number, then the translational and rotational (about short axis) drag coefficients affecting the spheroid are

$$
\begin{aligned}
& \gamma=6 \pi \eta b G, \\
& \gamma_{\theta}=6 \eta V G_{\theta},
\end{aligned}
$$

where $V$ is the volume of the spheroid and $G$ is the geometric factor that renders the ellipsoid different relative to the case of a sphere. The geometric factors for prolate spheroids diffusing in 3D are analytically derived from Perrin's equations [5]

$$
\begin{gathered}
G_{a}=\frac{8}{3} \frac{1}{\left[\frac{2 \phi}{1-\phi^{2}}+\frac{2 \phi^{2}-1}{\left(\phi^{2}-1\right)^{3 / 2}} \ln \left(\frac{\phi+\sqrt{\phi^{2}-1}}{\phi-\sqrt{\phi^{2}-1}}\right)\right]}, \\
G_{b}=\frac{8}{3} \frac{1}{\left[\frac{\phi}{\phi^{2}-1}+\frac{2 \phi^{2}-3}{\left(\phi^{2}-1\right)^{3 / 2}} \ln \left(\phi+\sqrt{\phi^{2}-1}\right)\right]},
\end{gathered}
$$

and $[3,12]$

$$
G_{\theta}=\frac{2}{3} \frac{\phi^{4}-1}{\phi\left[\frac{2 \phi^{2}-1}{\sqrt{\phi^{2}-1}} \ln \left(\phi+\sqrt{\phi^{2}-1}\right)-\phi\right]} .
$$

Here, $\phi=a / b$ is the ellipsoid aspect ratio. When $\phi=1$, then $G=G_{\theta}=1$ and Eq. (2) reduces to the translational and rotational Stokes laws for a sphere. Note also that Eqs. (2) and (3) are obtained using stick boundary conditions, valid when the particle is much larger than the fluid molecules $[13,14]$. In Fig. 1, Eq. (3) is plotted as a function of $\phi$ for $\phi$ less than 10. When the aspect ratio $\phi \gg 1$, Eqs. (1), (2a), (2b), and (3a)-(3c) yield

$$
D_{a}=\frac{k_{B} T \ln \phi}{2 \pi \eta a}, \quad D_{b}=\frac{k_{B} T \ln \phi}{4 \pi \eta a} .
$$

The ratio between these diffusion coefficients along long and short axes, i.e., $D_{a} / D_{b}=G_{b} / G_{a}$, increases monotonically from 1 to 2 as $\phi$ increases from 1 to infinity (in 3D). In quasi-2D, however, $D_{a} / D_{b}$ can be larger than 2 .

\section{EXPERIMENT}

The diffusion of micrometer size polymethyl methacrylate (PMMA) and polystyrene (PS) ellipsoids was measured in water confined between two glass walls. Both PS and PMMA ellipsoids are synthesized by the method described in Ref. [15]. Briefly, we placed $0.5 \%$ (by weight) PS spheres into a $12 \%$ (by weight) aqueous polyvinyl alcohol (PVA) solution residing in a Petri dish. After water evaporation, the PVA film was stretched at $130{ }^{\circ} \mathrm{C}$. The PS (or PMMA) spheres embedded in the film are readily stretched under these circumstances because their glass transition temperatures are below $130{ }^{\circ} \mathrm{C}$. After cooling to room temperature, the PVA was dissolved and ellipsoids obtained. Note that the initial PMMA or PS spheres must not be cross-linked, otherwise they cannot be stretched. We measured the size of ellipsoids by scanning electron microscopy (SEM) and by optical microscopy.

The ellipsoid solutions were cleaned and stabilized with 7 $\mathrm{mM}$ sodium dodecyl sulfate (SDS). The ellipsoids were not expected to have strong interactions with the glass surfaces because the solution ionic strength was more than $0.1 \mathrm{mM}$ and the Debye screening length for the particles was correspondingly less than $30 \mathrm{~nm}$. However, it is difficult to estimate the ionic strength accurately in a thin cell because the glass surfaces can release $\mathrm{Na}^{+}$ions [16]. Nevertheless, we found that the addition of $2 \mathrm{mM}$ salt to the solution did not induce a detectable change in particle diffusion coefficients. This observation suggests the double layers are not significantly affecting particle diffusion.

Glass surfaces of the sample cell were rigorously cleaned in a 1:4 mixture of hydrogen peroxide and sulfuric acid by sonication. Then the glass was thoroughly rinsed in deionized water and quickly dried with an air blow gun. Typically $0.3 \mu \mathrm{L}$ solution spread over the entire $1.8 \times 1.8 \mathrm{~mm}^{2}$ coverslip area and ellipsoids did not stick to the surfaces. Because the ellipsoid's gravitational height, $k_{B} T / m g$, is much larger than the cell thickness, $H$, the ellipsoids were readily suspended around midplane between the two walls. Finally, the cell was sealed with UV-cured adhesive (Norland 63).

We measure the wall separation by light interference. When the cell thickness is below a few micrometers, then the interference colors produced by reflections from the two inner surfaces of the sample walls in white light illumination can be observed by eye or in the reflection mode of the microscope [see Figs. 2(b) and 2(c)]. When the wall separation $H=0$, the effective light path difference is $\Delta l=\lambda / 2$ due to the $\pi$ phase shift of reflection at the lower surface. Thus all wavelength components of the white light yield a dark black color in interference at $H=0$. When $H>0$, the reflected light in the normal direction is a mixture of light with various wavelengths, and different wavelengths contribute with different weights to the observed color. White light interference from a wedge, for example, will yield bands of colors as in the Michel-Levy chart [17]. By comparing the experimentally observed color to the Michel-Levy chart, one can effec- 
(A)
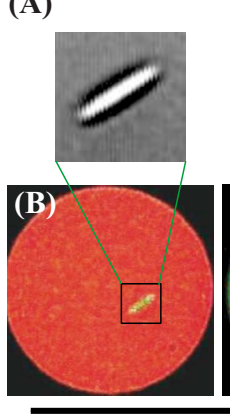

(C)
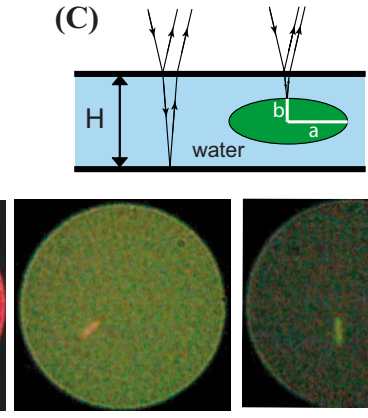

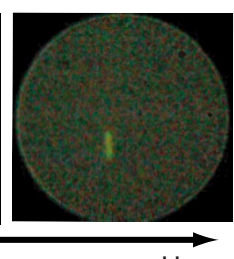

$\mathrm{H}$

FIG. 2. (Color online) (a) Bright field ellipsoid image in the transmission mode. (b) True interference color images of ellipsoid in the reflection mode of the microscope. The sample cell thickness increases from the left to the right. (c) Schematic of sample dimensions and the interference observation mode.

tively read out a corresponding $\Delta l$ and obtain $H=\Delta l /\left(2 n_{w}\right)$, where $n_{w}$ is the refractive index of water. In the Michel-Levy chart, the color starts from black at $\Delta l=0$ and changes from red to blue periodically with period $\Delta l=625 \mathrm{~nm}$. To avoid misreading the color by one or more periods, we established a reference thickness with either reference wedges or using dilute spacer spheres with known diameter between the glass slides. Color bands can shift slightly because the illumination light is not an ideal white light source. This error, however, should be less than $625 / 4 \mathrm{~nm}$, so that the error of $H$ is less than $\delta H \sim(625 \mathrm{~nm} / 4) /\left(2 n_{w}\right)=60 \mathrm{~nm}$. Furthermore, although the absolute value of $H$ may be subject to $\sim 60 \mathrm{~nm}$ uncertainty as described above, the relative values of different $H$ in one cell are more accurately determined ( $\sim 30 \mathrm{~nm}$ ) because we can easily distinguish more than eight different colors in one band including deep red, light red, orange, light orange, yellow, green, light green, blue, and violet. Our sample thickness typically had less than $20 \mathrm{~nm}$ variation in the central $1 \mathrm{~mm}^{2}$ area and only a 1-2 $\mu \mathrm{m}$ variation over the whole $18 \times 18 \mathrm{~mm}^{2}$ area. Thus, we could study the diffusion of ellipsoids at different $H$ in a single cell.

The interference between reflected light from the top inner surface of the sample wall and the ellipsoid's top surface gives rise to different colors (see Fig. 2) compared to the colors generated by interference of the top/bottom sample cell walls. Color changes due to variation in the separation between the top sample wall and the ellipsoid top surface are shown in the Fig. 2 as a function of $H$. As is the case with Newton's rings, the interference colors due to the two ellipsoid tips and the center of the ellipsoid were different. We generally found that the color only fluctuated near the two ellipsoid tips; the color was quite constant near the ellipsoid center. Thus the height fluctuation of the ellipsoids was measured to be very small $(<40 \mu \mathrm{m})$ and tumbling motions in the vertical plane were not strong $(<100 \mu \mathrm{m})$.

Particle motions observed by microscopy were recorded by a charge coupled device (CCD) camera to videotape at 30 frames/s. In the dilute suspension, only one ellipsoid was visible in the $640 \times 480$ pixel $^{2}=51.2 \times 38.4 \mu \mathrm{m}^{2}$ field of view under the $100 \times$ objective during a half-hour experiment. Typically, we defocus the objective slightly so that the

ellipsoid could be more accurately located along its long axis. The built-in 2D Gaussian fit function in interactive data language (IDL) was used to locate the center and orientation of the ellipse in each video frame. In practice, a small percent $(\sim 3 \%)$ of the frames failed to be correctly tracked. Without these frames, however, the trajectory breaks into short pieces and very long-time behavior becomes difficult to measure. Thus it is critical to recapture the lost frames. To capture these frames, we very slightly adjusted tracking parameters or image contrast in the code and then reanalyzed the images; after these corrections, roughly $3 \% \times 3 \%=0.09 \%$ of the frames remain incorrectly tracked. We then repeated this procedure iteratively until all $\sim 50000$ frames in one data set were correctly tracked.

The mean-square displacement (MSD) at time lag $t=0$ has a small nonzero intercept due to tracking errors. Thus we can estimate the spatial and angular resolutions from intercepts of their corresponding MSDs [18]. Using these procedures, we assign an orientation resolution of $1^{\circ}$ and spatial resolutions of 0.5 pixel $=40 \mathrm{~nm}$ along the particle's short axis and 0.8 pixel $=64 \mathrm{~nm}$ along its long axis because of the superimposed small tumbling motion [2].

From the image analysis, we obtained the trajectory of a particle's center-of-mass positions $\mathbf{x}\left(t_{n}\right)=\left[x\left(t_{n}\right), y\left(t_{n}\right)\right]$ in the laboratory frame and its orientation angle $\theta\left(t_{n}\right)$ relative to the $x$ axis at times $t_{n}=n(1 / 30)$ sec [see Fig. 1c of Ref. [2]]. We define each $1 / 30$-s time interval as a step. During the $n$th step, the particle's position changes by $\delta \mathbf{x}\left(t_{n}\right)=\mathbf{x}\left(t_{n}\right)-\mathbf{x}\left(t_{n-1}\right)$ and its angle by $\delta \theta\left(t_{n}\right)=\theta\left(t_{n}\right)-\theta\left(t_{n-1}\right)$. To obtain the drag coefficients along long and short axes, we need to convert the measured displacements from the fixed laboratory frame to the local body frame. Step displacements $\delta \widetilde{\mathbf{x}}_{n}$ relative to the local body frame and step displacements $\delta \mathbf{x}_{n}$ relative to the fixed laboratory frame are related via

$$
\left(\begin{array}{c}
\delta \tilde{x}_{n} \\
\delta \tilde{y}_{n}
\end{array}\right)=\left(\begin{array}{cc}
\cos \theta_{n} & \sin \theta_{n} \\
-\sin \theta_{n} & \cos \theta_{n}
\end{array}\right)\left(\begin{array}{c}
\delta x_{n} \\
\delta y_{n}
\end{array}\right),
$$

where $\theta_{n}=\left[\theta\left(t_{n-1}\right)+\theta\left(t_{n}\right)\right] / 2$ [see Fig. 1c of Ref. [2]]. In practice, choosing $\theta_{n}=\theta\left(t_{n-1}\right)$ or $\theta_{n}=\theta\left(t_{n}\right)$ has little effect on our results because $\theta$ barely changes during $1 / 30 \mathrm{~s}$.

\section{RESULTS AND DISCUSSION}

In both the laboratory and the body frame, MSDs are diffusive with $\left\langle[\Delta \widetilde{x}(t)]^{2}\right\rangle=2 D_{a} t, \quad\left\langle[\Delta \widetilde{y}(t)]^{2}\right\rangle=2 D_{b} t$, $\left\langle[\Delta x(t)]^{2}\right\rangle=\left\langle[\Delta y(t)]^{2}\right\rangle=\left(D_{a}+D_{b}\right) t \equiv 2 \bar{D} t, \quad$ and $\quad\left\langle[\Delta \theta(t)]^{2}\right\rangle$ $=2 D_{\theta} t[2]$. Here the brackets, \langle\rangle , denote data averaged over all possible time intervals in the whole trajectory of an ellipsoid. For example, an $N$-step total time trajectory (with 30 steps per second due to our frame rate of $30 \mathrm{~Hz}$ ) contains $N-30 t$ mean-square displacement data points for a correlation time interval of $t$ seconds. Figure 3 shows MSDs of a $2.4 \times 0.3 \times 0.3 \mu \mathrm{m}^{3}$ ellipsoid confined in an 846-nm-thick cell. All curves exhibit diffusive behavior and the diffusion coefficients, $D=\mathrm{MSD} /(2 t)$, shown in the figure are derived from the best fit lines. The slopes show that $D_{\theta}=0.161 \mathrm{~s}^{-1}, D_{a}=0.179 \mu \mathrm{m}^{2} / \mathrm{s}, D_{a}=0.044 \mu \mathrm{m}^{2} / \mathrm{s}$, and $D_{x}=D_{y}=\left(D_{a}+D_{b}\right) / 2$. 


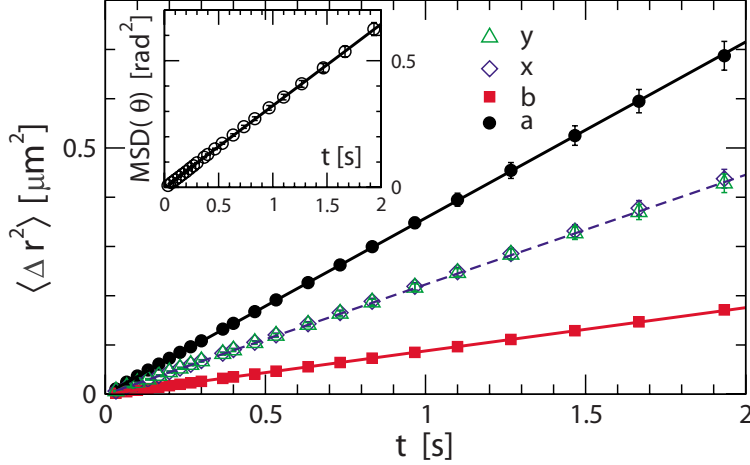

FIG. 3. (Color online) MSDs of a $2.4 \times 0.3 \times 0.3 \mu \mathrm{m}^{3}$ ellipsoid confined in an 846-nm-thick cell. The four lines arranged from top to bottom, respectively, are MSDs along $a, x, y$, and $b$ axes. Symbols represent experimental data and lines represent linear fits to these data. (Insets) Angular MSD (circles) and the best fit (line)

The error bars associated with each point in the figure, i.e., the standard deviations of the MSDs, were measured to be proportional to $t^{3 / 2}$. This can be understood from the probability theory. As an example, we derive the error of the total MSD, $(\Delta \mathbf{r})^{2}=(\Delta a)^{2}+(\Delta b)^{2}=(\Delta x)^{2}+(\Delta y)^{2} . \quad \Delta a$ and $\Delta b$ are statistically independent since $\gamma_{a}$ and $\gamma_{b}$ are constant independent of angle. Consequently, the variance $\sigma_{(\Delta \mathbf{r})^{2}}^{2}=\sigma_{(\Delta a)^{2}}^{2}+\sigma_{(\Delta b)^{2}}^{2}$. In probability theory [19], given two probability distribution functions (PDFs), $f_{\alpha}(x)$ and $f_{\beta}(x)$ for $\alpha$ and $\beta$ statistically independent, the joint PDF of their product is

$$
f_{\alpha \beta}(x)=\int_{-\infty}^{\infty} f_{\alpha}\left(x_{1}\right) f_{\beta}\left(x-x_{1}\right) d x_{1} .
$$

Since $\Delta a$ follows a Gaussian distribution [2] $f_{\Delta a}(x)$ $=\frac{1}{\sqrt{2 \pi} \sigma_{a}} e^{-x^{2} / 2 \sigma_{a}^{2}}$, the probability density function of $(\Delta a)^{2}$ is

$$
f_{(\Delta a)^{2}}(x)=\left\{\begin{array}{ll}
\frac{1}{\sqrt{2 \pi x} \sigma_{a}} e^{-x / 2 \sigma_{a}^{2}} & x \geqslant 0 \\
0 & x<0
\end{array} .\right.
$$

This distribution of $(\Delta a)^{2}$ was confirmed in our data. Hence the variance of $(\Delta a)^{2}$ is

$$
\begin{aligned}
\sigma_{(\Delta a)^{2}}^{2} & =\int_{-\infty}^{\infty} f_{(\Delta a)^{2}}(x) x^{2} d x \\
& =\int_{0}^{\infty} \frac{1}{\sqrt{2 \pi x} \sigma_{a}} e^{-x / 2 \sigma_{a}^{2} x^{2}} d x=3 \sigma_{a}^{4}=12 D_{a}^{2} t^{2} .
\end{aligned}
$$

Here, $t$ is the step time. Thus the standard deviation of $(\Delta \mathbf{r})^{2}$ is

$$
\frac{\sigma}{\sqrt{M}}=\frac{\sqrt{12 D_{a}^{2} t^{2}+12 D_{b}^{2} t^{2}}}{\sqrt{t_{t o t} / t}} \sim t^{3 / 2},
$$

where $M$ is the number of independent steps during the whole experimental time $t_{\text {tot }}$. (Note that the error bars of the figures in Ref. [2] were similarly calculated, albeit no theoretical explanation was given.)

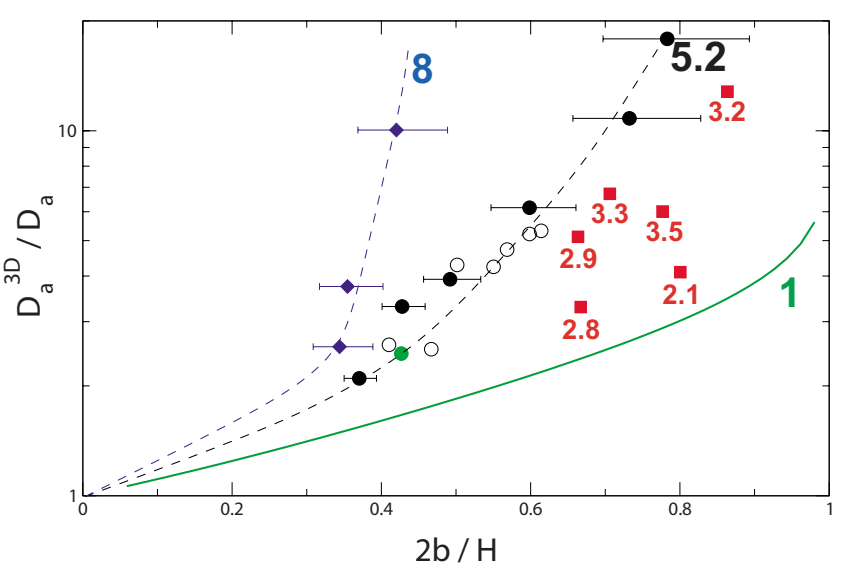

FIG. 4. (Color online) Ratio of theoretical 3D diffusion coefficient [5] along the ellipsoid long axis, $D_{a}^{3 \mathrm{D}}$, to the measured diffusion coefficient, $D_{a}$, for ellipsoids confined at $2 b / H$. Diamonds: $2.4 \times 0.3 \times 0.3 \mu \mathrm{m}^{3} \quad(\phi=8)$ ellipsoids; circles: $3.3 \times 0.635 \times 0.635 \mu \mathrm{m}^{3}(\phi=5.2)$ ellipsoids; solid circles: from samples with no added salt; open circles: from samples with $2 \mathrm{mM}$ added salt; green solid circle: from sample with bovine serum albumin (BSA) covered glass surfaces; squares: all other sampleslower aspect ratio spheroids with particle aspect ratios labeled below each data point. The accuracy of $2 b / H$ for these measurements is similar to other samples. Dashed curves: guides for the eyes. Solid curve $(\phi=1)$ : replot of the numerical prediction in Fig. 1 of Ref. [6] for a sphere strictly in the $H / 2$ midplane.

We repeated the experiments described above for different ellipsoids under different confinement conditions. From the slopes of their MSDs, we obtain $D_{a}, D_{b}$, and $D_{\theta}$ of different particles as a function of confinement condition as shown in Figs. 4-6, respectively. Specifically, the normalized quantities, $D_{i}^{3 \mathrm{D}} / D_{i}=\gamma_{i} / \gamma_{i}^{3 \mathrm{D}}$, for $i=a, b, \theta$, are plotted as a function of increasing confinement, $2 b / H$. Here the $3 \mathrm{D}$ normalization constants, $D_{i}^{3 \mathrm{D}}$ (alternatively, $\gamma_{i}^{3 \mathrm{D}}$ ), are calculated from Eqs. (2) and (3). (Note that these figures contain the same infor-

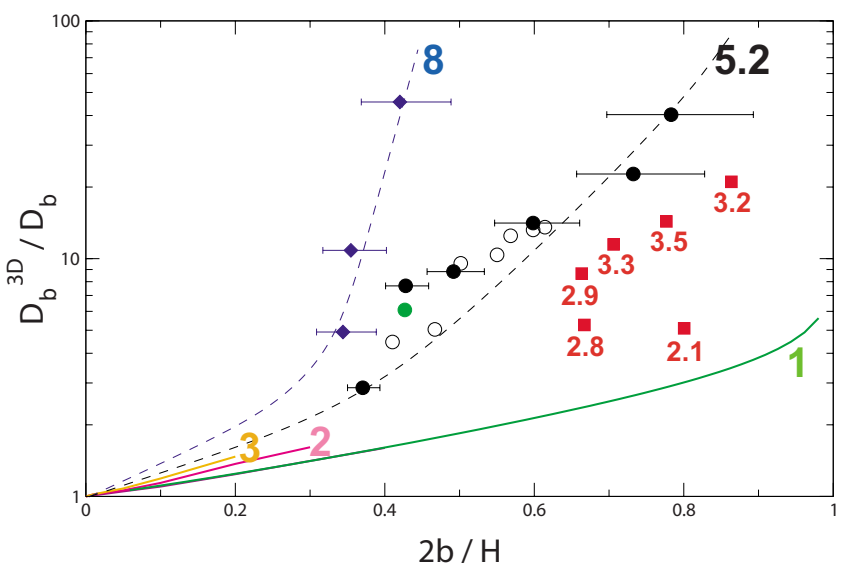

FIG. 5. (Color online) Ratio of theoretical 3D diffusion coefficient [5] along the ellipsoid short axis, $D_{b}^{3 \mathrm{D}}$, to the measured diffusion coefficient, $D_{b}$, for ellipsoids confined at $2 b / H$. Symbols are the same as those in Fig. 4. Solid curves from left to right: theoretical weak confinement predictions [5] for aspect ratios $a / b=3,2$ and numerical result [6] for aspect ration $a / b=1$ ranging over both weak and strong confinement regimes. 


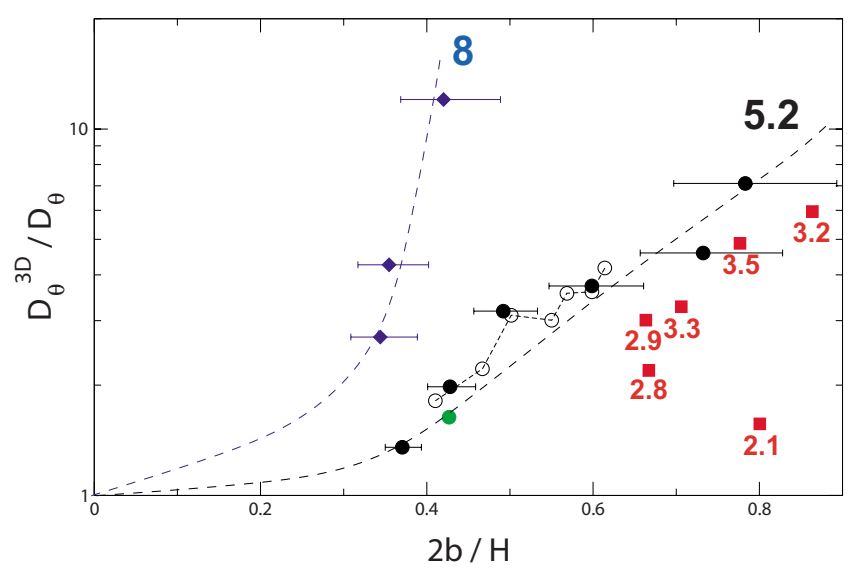

FIG. 6. (Color online) Ratio of theoretical rotational diffusion coefficient $[3,12] D_{\theta}^{3 \mathrm{D}}$ to the measured diffusion coefficient, $D_{\theta}$, for ellipsoids confined at $2 b / H$. Symbols are the same as those in Fig. 4.

mation as figures S3, S4, and S5 from the supplementary online material of Ref. [2].)

Notice that $2 b / H=0$ corresponds to the $3 \mathrm{D}$ limit wherein $D^{3 \mathrm{D}} / D=1$. As expected, hydrodynamic drag increased and the diffusion coefficients correspondingly decreased as the confinement became stronger. The larger positive slopes exhibited by the more needlelike spheroids are indicative of motions more sensitive to confinement. Furthermore, the slopes of the same ellipsoids in Fig. 5 are larger than those in Fig. 4, indicating that diffusion along the ellipsoid short axis is more strongly affected by the confinement than diffusion along the long axis. Limited comparisons to all available analytical and numerical predictions (i.e., the solid curves in Figs. 4 and 5) suggest that our data exhibit the generally expected trends with increasing confinement. Note that the solid curves of analytical and numerical predictions in Figs. 4 and 5 are for particles forced to sit in the $z=H / 2$ midplane. In real experiments, the measured drag is an average over different $z$. In our experiments, there are no detectable interference color changes at the centers of ellipsoids. Consequently $z$ fluctuations are less than $\sim 50 \mathrm{~nm}$, i.e., $\sim H / 20$. Numerical results in Ref. [6] indicate that the drag on a sphere at $H / 3$ is very close $(<10 \%)$ to the drag at $H / 2$. Thus the $z$ fluctuations of our ellipsoids should have only a very small effect on the reported particle drag.

Another question that our data hold potential to explore concerns the effect of electric double layers on ellipsoid diffusion. The electric double layers around charged particles in suspension increase their hydrodynamic diameter and slow down diffusion, especially rotational diffusion because rotational drag is proportional to the volume rather than the length of the ellipsoid. This effect in rotational diffusion has been observed with depolarized dynamic light scattering in the regime where ionic concentration was low and spheroids small [20]. In our systems, such effects might be expected to be small due to the high ionic strength of the suspension. As can be seen in Figs. 4-6, diffusion coefficients for the samples with $2 \mathrm{mM}$ added salt and no added salt are indistinguishable. The analytical Eq. (3) shows that the $6.9 \mathrm{~nm}$ screening layer of $2 \mathrm{mM}$ solution will lower the 3D diffusion coefficients by less than $2 \%$.

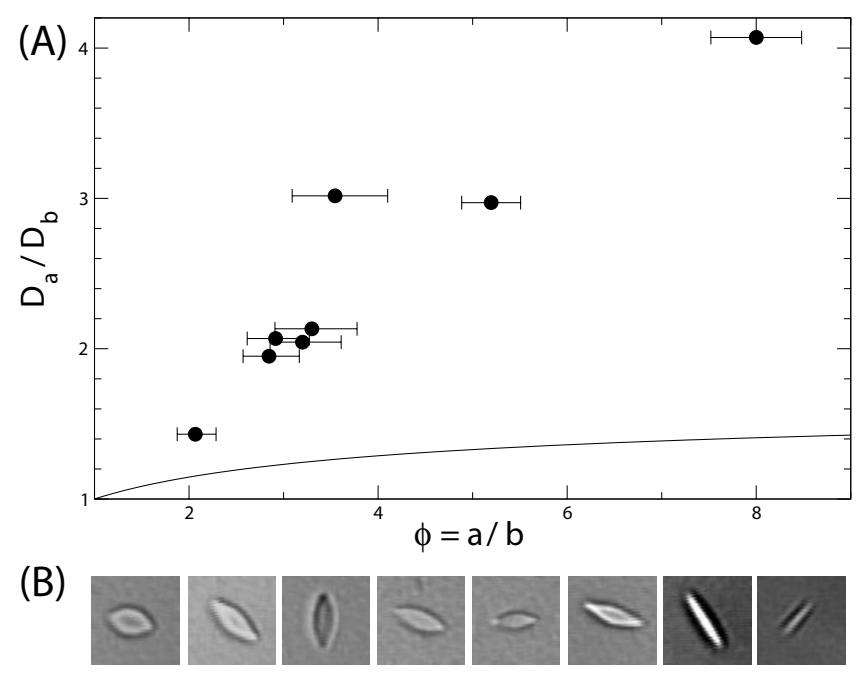

FIG. 7. (a) Ratio of diffusion coefficients $D_{a} / D_{b}$ vs aspect ratio $\phi=a / b$. Seven of eight data points were taken for $2 b / H \simeq 0.8$; the data point at $a / b=8$ was taken with $2 b / H \simeq 0$. 4 . Solid curve: theoretical curve for $3 \mathrm{D}$. (b) Microscope images of the corresponding eight particles as a function of aspect ratio (increasing from left to right). The dimensions of each particle from left to right: $[2 a, 2 b]=[2.48,1.2],[2.96,1.04],[2.8,0.96],[2.88,0.9]$, $[2.64,0.8],[3.12,0.88],[3.3,0.635]$, and $[2.4,0.3] \mu \mathrm{m}$.

Finally, Fig. 7 shows the impact of aspect ratio on the translational diffusion anisotropy ratio $D_{a} / D_{b}$. Here, it is evident that diffusion in quasi-2D is quite different from diffusion in $3 \mathrm{D}$. For $3 \mathrm{D}, D_{a} / D_{b}$ asymptotes to 2 at large aspect ratio, as shown by the solid theoretical curve. For quasi-2D, on the other hand, $D_{a} / D_{b}$ grows very rapidly with increasing aspect ratio. Since we expect the stick boundary condition to hold in this system, the observation that $D_{a} / D_{b}=\gamma_{b} / \gamma_{a}>2$ is purely due to confinement.

A schematic to qualitatively capture this basic effect is given in Fig. 8. Imagine fluid flowing past the ellipsoid. In $3 \mathrm{D}$, the fluid flow pathways will be displaced by distances of order $2 b$ in order to "go around" the ellipsoid. This fluid flow displacement is the approximately the same, whether the spheroid is orientated either parallel or perpendicular to the flow, and therefore $\gamma_{a}$ and $\gamma_{b}$ are thus comparable. In 2D, however, the fluid flow displacement pathway is approximately $2 b$ (or $2 a$ ) when the spheroid is oriented parallel (perpendicular) to the flow, so that $\gamma_{b} / \gamma_{a}$ diverges with increasing $a / b$. This very simple qualitative picture also explains our observation that diffusion along the ellipsoid short axes is more strongly affected by the confinement than diffusion

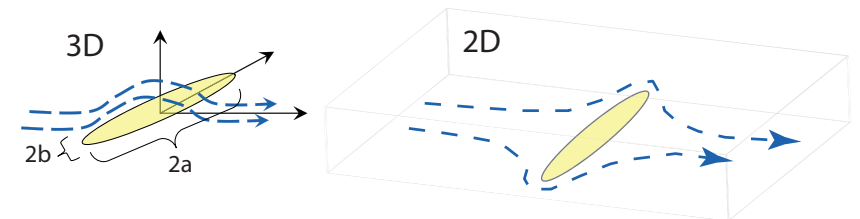

FIG. 8. (Color online) Schematic of fluid flow around an ellipsoid perpendicular to the flow. For the drag along short axes, fluid can flow around $2 b$ in $3 \mathrm{D}$, but has to flow around long axis $2 a$ in $2 \mathrm{D}$. 
along the long axis. The rotational diffusion is also not so strongly affected by the confinement (e.g., similar to $D_{a}$ ) because "easy" flow pathways exist to accommodate this ellipsoid motions. Finally, we note that in quasi-2D confinement, $D_{a} / D_{b}$ increases with increasing aspect ratio and should eventually saturate according to numerical calculations [6] at a value much larger than 2 because some fluid will always "leak" between the particle and walls.

In summary, we have found that the anisotropic drag coefficients for ellipsoid diffusion substantially increase when the ellipsoids are strongly confined between parallel plates, especially diffusion along their short axes. In the future, many questions about these systems will be exciting to explore, including the effects of neighboring ellipsoids and the effects of other confinement geometries. For example, quasione-dimensional (1D) confinement of an ellipsoid will align the ellipsoid along the diffusion direction. This effect may compensate drag from the boundaries and lead to an optimal diameter for ellipsoid diffusion speed in a quasi-1D cylinder.

\section{ACKNOWLEDGMENTS}

We thank Jerzy Blawzdziewicz for providing us with the theoretical curves at $\phi=1$ in Figs. 4 and 5. We also thank Tom Lubensky for useful conversations. This work was supported by the NSF-MRSEC under Grant No. DMR-0520020 and partially by the NSF under Grant No. DMR-0804881.
[1] P. G. Saffman and M. Delbruck, Proc. Natl. Acad. Sci. U.S.A. 72, 3111 (1975).

[2] Y. Han, A. Alsayed, M. Nobili, J. Zhang, T. C. Lubensky, and A. G. Yodh, Science 314, 626 (2006).

[3] F. Perrin, J. Phys. Radium 5, 497 (1934).

[4] F. Perrin, J. Phys. Radium 7, 1 (1936).

[5] J. Happel and H. Brenner, Low Reynolds Number Hydrodynamics (Kluwer, Dordrecht, 1991).

[6] S. Bhattacharya, J. Blawzdziewicz, and E. Wajnryb, J. Fluid Mech. 541, 263 (2005).

[7] B. Lin, J. Yu, and S. A. Rice, Phys. Rev. E 62, 3909 (2000).

[8] E. R. Dufresne, D. Altman, and D. G. Grier, Europhys. Lett. 53, 264 (2001).

[9] D. Mukhija and M. J. Solomon, J. Colloid Interface Sci. 314, 98 (2007).

[10] M. Kim, S. M. Anthony, and S. Granick, Soft Matter 5, 81 (2009).
[11] B. Bhaduri, A. Neild, and T. W. Ng, Appl. Phys. Lett. 92, 084105 (2008).

[12] S. Koenig, Biopolymers 14, 2421 (1975).

[13] C. M. Hu and R. Zwanzig, J. Chem. Phys. 60, 4354 (1974).

[14] D. R. Bauer, J. I. Brauman, and R. Pecora, J. Am. Chem. Soc. 96, 6840 (1974).

[15] C. C. Ho, A. Keller, J. A. Odell, and R. H. Ottewill, Colloid Polym. Sci. 271, 469 (1993).

[16] J. C. Crocker, Ph.D. thesis, The University of Chicago, 1996.

[17] N. H. Hartshorne and A. Stuart, Crystals and the Polarising Microscope (Edward Arnold Ltd., London, 1970).

[18] J. C. Crocker and D. G. Grier, J. Colloid Interface Sci. 179, 298 (1996).

[19] S. Ross, A First Course in Probability (Pearson Prentice Hall, New Jersey, 2006)

[20] H. Matsuoka, H. Morikawa, and H. Yamaoka, Colloids Surf., A 109, 137 (1996). 